\title{
Association of CYP2D6*4 genetic polymorphism on the metabolism of Donepezil with Alzheimer's disease in Indian population
}

\author{
Vivekanandhan Subbiah ${ }^{*}$, Sonali Nirmal ${ }^{1}$, Manjari Tripathi ${ }^{2}$, Rajesh Sagar ${ }^{3}$ \\ From $1^{\text {st }}$ International Congress on Neurobiology and Clinical Psychopharmacology and European Psychia- \\ tric Association Conference on Treatment Guidance \\ Thessaloniki, Greece. 19-22 November 2009
}

\section{Background}

Alzheimer's disease (AD) is the most common adult form of dementia.1 It is an age-associated neurodegenerative disorder pathologically characterized by the abnormal accumulation of intracellular neurofibrillary tangles and extra cellular amyloid plaques in selected brain regions. Donepezil is a cholinesterase inhibitor currently being used in the treatment of Alzheimer's disease is metabolized via CYP2D6 enzymes. The present study was undertaken to investigate CYP2D6*4 polymorphism on the serum concentration of Donepezil with responders and non-responders to Alzheimer's patients.

\section{Materials and methods}

40 Alzheimer's patients with responders to Donezepil drug and 40 Alzheimer's patients with non-responders to donezepil drug were investigated for CYP2D6*4 polymorphism using polymerase chain reaction - restriction fragment polymorphisms (PCR-RFLP). Allele frequencies were derived from genotypic data. Drug responders non-responders' comparisions were made using ChiSquare tests. Deviations from the Hardy - Weinberg equilibrium were also tested. Drug levels of Donezepil were determined using HPLC method.

\section{Results}

The CYP2D6*4 Polymorphism was seen to be in Hardy - Weinberg equilibrium and showed significant allelic association and genotypic association between responders and non-responders of donezepil. Genotypic:
$\mathrm{P}=0.05 ; \mathrm{OR}=0.39(0.13-1.15)$, Allelic: $\mathrm{P}=0.008$; $\mathrm{OR}=2.79(1.20-6.58)$.

\section{Conclusions}

Our finding suggest that the CYP2D6 * 4 genetic polymorphism may be associated with the individual differences in donezepil metabolism. An individualized dosage regimen design incorporating such genetic information would help to increase the clinical efficacy of donezepil in Alzheimer's patients.

\section{Author details \\ 'Department of Neurobiochemistry, All India Institute of Medical Sciences, New Delhi, Delhi, India. ²Department of Neurology, All India Institute of Medical Sciences, New Delhi, Delhi, India. ${ }^{3}$ Department of Psychiatry, All India Institute of Medical Sciences, New Delhi, Delhi, India.}

Published: 22 April 2010

\section{Reference}

1. Cacabelos: Pharmacogenomics and therapeutic prospects in Alzheimer's disease. Mol Diagn Ther 2007, 11(6):385-405.

\section{doi:10.1186/1744-859X-9-S1-S142}

Cite this article as: Subbiah et al:: Association of CYP2D6*4 genetic polymorphism on the metabolism of Donepezil with Alzheimer's disease in Indian population. Annals of General Psychiatry 2010 9(Suppl 1): S142. 